points, including at mother-infant pair (MIP) clinics, nutrition rehabilitation units (NRU) and paediatric wards.

Methods An analysis of the 2015 databases of Community Management of Acute Malnutrition (CMAM), Laboratory Information Management System (LMIS) and EID at point-of-care (EID POC) was conducted to identify optimal entry points for identifying HIV-infected children. A chi-square test was used to determine differences between groups.

Results A total of 7629 children below 5 years of age were admitted in NRUs; $60 \%$ were tested for HIV and 17\% were HIV-infected. The EID POC database showed that most (50\%) of the children identified from the inpatient paediatric ward were HIV-infected as compared to $2.5 \%$ in MIP clinics and $11 \%$ in outpatient paediatric wards. A chi-square test of significance shows that the HIV positivity varies between entry points (chi-square value $=182.34$, with 2 degrees of freedom and p-value $<0.001)$. The LMIS database showed that $45 \%$ of children identified in the paediatric ward were HIV-infected compared to $30 \%$ of children identified via NRUs and $4 \%$ in MIP clinics. A chi-square test of significance shows that HIV positivity varies between entry points (chi-square value $=597.83$, with 6 degrees of freedom and p-value $<0.001)$.

Conclusions High yield of HIV positivity in children was found in the paediatric wards and NRUs as opposed to MIP and outpatient wards. Targeting EID POC testing to these settings can reduce infant mortality and morbidity as HIV-infected children will be identified and initiated on treatment more quickly.

\title{
PA-056 NEED FOR REANALYSIS OF CURRENT TESTING OF HIV-EXPOSED INFANTS
}

Emmanuel Saka, ${ }_{1}$ Zex Kalipalire, ${ }^{1}$ Ravikiran Bhairavabhotla, ${ }^{2}$ Dalitso Midiani, ${ }_{1}^{3}$ Judith Sherman'. ' $U N I C E F$, Malawi; ${ }^{2}$ UNICEF, United States of America; ${ }^{3}$ HIV \& AIDS Department, Ministry of Health, Malawi

10.1136/bmjgh-2016-000260.89

Background In 2015, 12\% of HIV-exposed infants in Malawi received an early infant diagnostic test (EID) in the first two months of life. Provider-initiated testing and counselling (PITC) is recommended as a standard component of comprehensive clinical management for inpatients and outpatients in entry 